\title{
Salt reduction in China: a state-of-the-art review
}

This article was published in the following Dove Press journal:

Risk Management and Healthcare Policy

22 February 2017

Number of times this article has been viewed

\section{Shuai Shao ${ }^{1,2}$ \\ Yechu Hua ${ }^{3}$ \\ Ying Yang ${ }^{4}$ \\ Xiaojuan Liu $^{5}$ \\ Jingruo $\mathrm{Fan}^{6}$ \\ An Zhang ${ }^{7}$ \\ Jingling Xiang ${ }^{8}$ \\ Mingjing $\mathrm{Li}^{9}$ \\ Lijing L Yan ${ }^{1,2}$}

'Global Health Research Center, Duke Kunshan University, Kunshan, jiangsu, China; ${ }^{2}$ Duke Global Health Institute, ${ }^{3}$ School of Economics, Duke University, Durham, NC, USA; ${ }^{4}$ Department of Public Health, Wuhan University, Wuhan, Hubei, ${ }^{5}$ Department of Biostatistics, School of Public Health, Shandong University, Jinan, Shandong, China; ${ }^{6}$ Department of Accounting, School of Business and Management, The Hong Kong University of Science and Technology, Kowloon, Hong Kong; ${ }^{7}$ Department of Statistics and Applied Probability, National University of Singapore, Singapore; ${ }^{8}$ School of Business, Sichuan University, Chengdu, Sichuan, ${ }^{9}$ Department of Public Health, School of Public Health and Management, Chongqing Medical University, Chongqing, China

Correspondence: Lijing L Yan Global Heath Research Center, Duke Kunshan University, 8 Duke Avenue, Kunshan, Jiangsu Province 215316, China Tel +86 5I 236657057

Fax +8651257728899

Email Lijing.yan@duke.edu
Objective: This study aimed to reveal the latest evidence on salt reduction initiatives in China in order to identify the contextual cost-effective interventions, as well as the barriers encountered during China's long march to reach its population salt reduction goal.

Background: Population-based salt reduction has been considered as one of the most costeffective strategies in the world for the prevention and control of noncommunicable diseases. China, along with its sustained economic growth, faces increasing burdens from chronic diseases such as cardiovascular and kidney diseases. With policy support and cross-sector collaboration, various salt reduction initiatives have been adopted in China in order to reduce such dietary risk, especially since the beginning of this millennium.

Methods: This study conducted structured literature reviews in both English and Chinese databases and synthesized the latest evidence on the association of salt intake and health, as well as salt intake among Chinese and population-based salt reduction strategies in China and around the world.

Findings: Dietary salt restriction has been found to contribute to the reduction of blood pressure among both the normotensives and hypertensives bringing associated reduced disease burdens and great public health benefits. With gender, ethnic, and regional variations, salt intake levels in the population in China are well above the recommended threshold and physiological need. Admittedly, excessive salt intake precipitates the high prevalence of hypertension and cardiovascular disease among the Chinese. Considering that the majority of the dietary salt is added during cooking in China, salt substitutes, salt restriction tools, and health education are the most common salt reduction initiatives with varying levels of effectiveness and acceptability among the Chinese population.

Implication: Overwhelming evidence is in support of a well-coordinated nationwide salt restriction initiative as a key public health strategy for the prevention and control of hypertension and its related diseases. Orchestrated efforts from the government, industries, academia, health professionals, and the general public are required to achieve China's long-term goal for salt reduction.

Keywords: chronic disease, hypertension, salt reduction, health policy, Chinese population

\section{Introduction}

Salt has long been included as the quintessential component of the human diet. Although crucial to most of the human physiological processes, excessive salt intake has been found to be associated with raised blood pressure (BP) and heightened risks of many chronic diseases including cardiovascular and kidney diseases. 
Ranked as the most common risk factor globally, high BP is responsible for 174 million disability-adjusted life years (DALYs) every year. ${ }^{1}$ The latest Global Burden of Disease Study 2013 (GBD 2013) ${ }^{2}$ indicated that 17.3 million people die from cardiovascular diseases annually, accounting for nearly one-third of the total deaths worldwide. Approximately $53 \%$ of DALYs related to ischemic heart disease, which is the most common type of cardiovascular diseases and the leading cause of death in the world, ${ }^{2}$ were attributable to high BP. ${ }^{3}$ Meanwhile, more than one-quarter of the 1 million annual deaths from chronic kidney diseases were due to hypertension. ${ }^{2}$

China bears a significant proportion of hypertensionrelated global mortality and morbidity. The GBD 2013 study indicated that $\sim 20 \%$ of total Chinese DALYs are related to cardiovascular diseases. ${ }^{4}$ Of the 230 million Chinese diagnosed with such diseases, 200 million have high BP and 7 million suffer from stroke. As aging gains more momentum in China, combined with population growth, it is estimated that the number of cardiovascular disease events will increase by $>50 \%$ by $2030 .^{5}$

Recognizing hypertension as a major threat to public health and the cost-effectiveness of population-based salt reduction strategies to reduce hypertension, the World Health Organization (WHO) has called for all member states to achieve a $30 \%$ relative reduction in mean population salt/ sodium intake by $2025 .{ }^{6}$ The present study aims to examine the scientific basis of such initiatives and synthesize the current salt intake and salt reduction initiatives in selected countries with a focus on China.

\section{Methods}

This study conducted structured literature reviews in both English and Chinese databases and synthesized the latest evidence on the association of salt intake and health, as well as salt intake among Chinese and population-based salt reduction strategies in China and around the world.

Separate structured literature searches were conducted on four subthemes:

1. Salt and health

2. Salt intake in China

3. Salt reduction in the world

4. Salt reduction in China.

The relationship of the four key dimensions of this review (health, China, salt, and reduction) is illustrated in a Venn diagram (Supplementary material A1). Various keywords were further developed for each dimension. Specific English and
Chinese keywords are listed in Supplementary materials A2 and A3, respectively. Time period was set from 2000 to 2016.

For salt and health dimension, given the extremely extensive evidence on hand, we restricted our search to systematic review articles in English databases (PubMed, EMBASE, and Cochrane) only. Meanwhile, search was conducted in the same three English databases for the topic of salt reduction in the world.

For salt intake in China and salt reduction in China dimensions, searches were conducted based on the aforementioned keywords using Boolean combinations in both English (PubMed, EMBASE, and Cochrane) and Chinese databases (WanFang and CNKI). Studies were selected for inclusion based on their information about study design, ascertainment of intervention, and use of standard definition of main outcome measures, attrition rates, and sample sizes. Review articles are also included after quality assessment.

Grey literature search was conducted in the popular Chinese search engine Baidu using similar keywords in Chinese characters. Reference lists of key references were further explored to obtain any relevant information.

\section{Findings \\ Salt and health}

Hypertension is a common chronic condition susceptible to the interplay between genetic and environmental factors, with salt intake exerting significant physiological influence. The sensitivity of BP to salt intake was documented as early as in $1904^{7}$ with more recent evidence indicating the association between high salt intake and heightened risk of hypertension and stroke events. ${ }^{8,9}$

In addition to the evidence from observational studies, research from interventional studies has convincingly demonstrated the relationship between salt intake and BP. Dietary salt restriction has been found to contribute to the reduction of BP ${ }^{8,10,11}$ among the normotensives, ${ }^{12-14}$ hypertensives, ${ }^{12-16}$ and people with chronic kidney disease. ${ }^{17}$ The latest systematic review in 2016 comprising 123 randomized trials involving 613,815 participants showed a $13 \%$ reduction in all-cause mortality associated with each decrease of $10 \mathrm{mmHg}$ in systolic blood pressure (SBP), ${ }^{18}$ indicating the public health benefits of a salt-restricted diet through BP reduction. Furthermore, a modest and long-term reduction in salt intake is predicted to reduce stroke mortality ${ }^{14}$ as well as to decrease proteinuria possibly translating to lower the incidence of end-stage kidney disease. ${ }^{17}$

Nevertheless, there are controversies regarding the potential health effect of "inadequate" salt intake. One systematic review reported increase in plasma renin, plasma aldosterone, 
plasma adrenaline, plasma noradrenaline, cholesterol, and triglyceride with reduced sodium intake, ${ }^{12}$ whereas a metaanlaysis in 2013 found no adverse effect on blood lipids, catecholamine levels, or renal function in studies of more than 4 weeks duration and with moderate change in sodium intake. ${ }^{8}$

The latest pooled analysis on sodium excretion in 133,118 individuals from $>49$ countries by Mente et al ${ }^{19}$ showed that cardiovascular disease risks were heightened with low sodium intake ( $<3 \mathrm{~g} /$ day) irrespective of hypertension status. Furthermore, excessive sodium intake ( $>7 \mathrm{~g} /$ day) was found to be associated with increased risk of cardiovascular diseases among hypertensive patients only. Such findings, if substantiated, would overturn the rationale of population-based salt reduction target, currently set at $5 \mathrm{~g}$ /day by the WHO. ${ }^{6}$ The study, along with the publishing journal The Lancet quickly drew criticism from leading academics ${ }^{20,21}$ and the World Action on Salt and Health (WASH). ${ }^{22}$ Its main methodological flaws included using a single spot urine excretion rather than the golden standard of $24 \mathrm{~h}$ urinary sodium excretion, ${ }^{20,22}$ the failure to avail all conditions satisfying Hill's criteria in order to establish casual association between low sodium intake and cardiovascular diseases event ${ }^{21}$ and the inherent issue of reverse mortality associated with the study design. ${ }^{22}$

Polarizing opinions from different studies with different methods of strength are not uncommon in science. The difficulty in accurate measurement of sodium, confounding commonly related with dietary variables along with the unfeasibility of constructing fully randomized trials makes the subject of population salt/sodium reduction a controversial public health matter. ${ }^{21} \mathrm{~A}$ new trial is currently ongoing in rural China to shed light on the direct effects of salt substitute (sodium reduction and potassium enrichment) on fatal and nonfatal stroke. ${ }^{23}$ While we await results from more rigorous research to understand the full effect of salt reduction on human health, paradoxical findings on salt intake at the lower end of the spectrum should not overturn the evidence-based public health message to reduce excessive salt intake.

Indeed, in the case of China, the INTERMAP study suggested that the difference in sodium intake between the north and south could explain about one-third of the north-south difference in SBP, supporting the notion of higher salt intake as the major contributor to the high prevalence of hypertension in China. ${ }^{24}$ As early as in 1982 , Chinese scholars have identified the relatively higher hypertension prevalence in Northern China compared to Southern China. ${ }^{25}$ The latest evidence includes a systematic review and meta-regression analysis in $2014 .{ }^{26}$ Based on this study, the age-standardized prevalence of hypertension was $28.3 \%$ in the northeast,
$30.4 \%$ in the middle north, $23.6 \%$ in the north-west, $23.3 \%$ in the east, $16.2 \%$ in the middle south, and $19.9 \%$ in the southwest China. ${ }^{26}$ Higher prevalence of heart failure ${ }^{27}$ and stroke ${ }^{28}$ were also found in northern China compared to the south.

A 2011 Lancet report initiated by the WHO and produced in collaboration with $\sim 100$ scientists included dietary salt reduction as one of the five priority interventions for member states to address the noncommunicable disease crisis. The report also estimated the cost of implementing mass-media campaigns and voluntary action by food industry to reduce dietary salt consumption to be at $\$ 0.05$ per person per year for China. ${ }^{29}$

The cost-effectiveness of salt reduction initiatives has been examined in other simulation studies. ${ }^{30,31}$ Based on a new meta-analysis comprising 107 randomized interventions and the Global Burden of Disease Study 2010, the NUTRICODE group found that in 2010, 191,451 deaths in China can be attributed to a daily sodium intake of $>2 \mathrm{~g}$, whereas $25.4 \%$ of total cardiovascular deaths in China result from such excessive daily sodium intake. ${ }^{30}$

Measured in terms of annual cardiovascular diseases events averted, quality-adjusted life years (QALYs) gained, cardiovascular diseases treatment costs saved, and the relative reductions in rates of cardiovascular disease incidence and mortality, Wang et $\mathrm{al}^{31}$ applied the Cardiovascular Disease Policy Model in 2015 to simulate the effect of reducing mean dietary salt intake and found that by reducing the salt intake to $9 \mathrm{~g}$ /day over a 10-year period in China, 197,000 annual cardiovascular events could be prevented while a $2.5 \%$ reduction in cardiovascular mortality could be anticipated. Furthermore, 303,000 annual QALYs could be gained and 1.4 billion international dollars (Int\$) could be saved. Lowering the target to $6 \mathrm{~g}$ /day could approximately double these benefits. The focus of dietary salt reduction strategies should be on hypertensive adults as three-quarters of the benefits above would be derived from them. ${ }^{31}$

In the following sections, first recent studies on the level of salt intake in China are summarized. After briefly introducing salt reduction strategies in selected countries, such strategies in contemporary China are focused. The review ends with policy and practice recommendations for reducing salt intake to improve health in China.

\section{Salt intake in China}

Long been recognized as the "king of all flavors," salt has played an essential role in the preparation and preservation of Chinese food for thousands of years. Studies on salt intake in China at the national, regional (provincial), and local (city) 
level in the past 16 years were reviewed and summarized. Sodium intake estimated from multiple-day 24-h urinary sodium excretion is regarded as the gold standard for the measurement of salt intake. ${ }^{32}$ However, it is harder to implement and more time consuming and places higher burden on participants than some other methods, such as single spot urinary excretion, dietary recall, or weighed food record. This review first describes the results from a limited number of studies based on 24-h urinary data and then summarizes the studies using other methods to estimate salt intake. A detailed description of salt intake levels throughout China can be found in Supplementary material B.

Using the 24-h urinary sodium excretion method, the INTERMAP study that China participated in the 1990s showed the average sodium intake from three sites was $3990 \mathrm{mg} /$ day $(10.1 \mathrm{~g} /$ day salt $)$ varying from $4733 \mathrm{mg} /$ day $(12.0 \mathrm{~g} /$ day salt $)$ in northern China (Beijing and Shanxi) to $2491 \mathrm{mg} /$ day (6.3 g/day salt) in southern China (Guangxi) ${ }^{24}$

Gender, ethnicity, and regional variations were further explored using the 24-h urinary sodium excretion method. Men were found to consume more salt ( $12 \mathrm{~g} /$ day) than women ( $10.3 \mathrm{~g}$ /day) in 11 locations including Guiyang, Guangzhou, Shanghai, Beijing, Shijiazhuang, and Taipei across China during the CARDIAC study. ${ }^{33}$ In terms of ethnicity variation, Tibetans (14.8 g/day) and Kazaks ( $12.5 \mathrm{~g} /$ day) consume more salt than Han (11.3 g/day) and Uygur (10.2 g/day). ${ }^{34}$ One 2003 study conducted in Beijing, Shanghai, and Guangxi also indicated that northern Chinese tend to eat more salt ( $>14 \mathrm{~g} /$ day) than southern Chinese ( $<9 \mathrm{~g} /$ day $)$ and men demonstrate consistently higher salt (8.8-17.2 g/day) intake compared to women (7.5-14.6 g/day) ${ }^{35}$ Regional studies using the 24-h urinary method have revealed the average salt intake to be $11 \mathrm{~g}$ /day in Jiangsu Province, ${ }^{36} 13.9 \mathrm{~g}$ /day in Shandong Province, ${ }^{37}$ and $11.8 \mathrm{~g} /$ day in the city of Yantai, Shandong Province. ${ }^{38}$

In the past decades, results from a growing number of published studies have suggested that most of China's population exceed the recommended daily salt consumption ( $5 \mathrm{~g} /$ day) set by the WHO. According to the 2002 Chinese Nutrition and Health Condition Survey, the nationwide salt intake is $10.7 \mathrm{~g} /$ day based on the dietary record from three consecutive days..$^{39} \mathrm{~A}$ recent study on 6,072 adults from 12 of China's mainland provinces found the mean salt consumption to be $9 \mathrm{~g} /$ day, which is based on laboratory-analyzed food sample and household dietary measurement. ${ }^{40}$ Average salt intake in Beijing was found to be $15.2 \mathrm{~g}$ /day for adults, $11.0 \mathrm{~g}$ /day for children, and $10.2 \mathrm{~g} /$ day for senior citizens. ${ }^{41}$ Salt intake in
Zhejiang province was found to be $10.5 \mathrm{~g} /$ day in $2011^{42}$ and $8.91 \mathrm{~g} /$ day in $2015 .{ }^{43}$ Daily salt intake was estimated to be $7.63 \mathrm{~g}$ in Fujian province, ${ }^{44} 7.6 \mathrm{~g}$ in Guangdong province, ${ }^{45}$ and $10.3 \mathrm{~g}$ in Hunan province. ${ }^{46}$ There is also a pattern of higher salt intake from in the western region $(12.5 \mathrm{~g} /$ day $)$ than the eastern region ( $8.6 \mathrm{~g} /$ day $)$ with rural residents taking in more salt $(11.5 \mathrm{~g} /$ day $)$ than their urban counterparts $(9.1 \mathrm{~g} /$ day $) .{ }^{47}$ As to occupational differences, farmers were found to consume more salt than industrial workers. ${ }^{48}$

Encouragingly, salt intake was found to have declined from 12.7 to $8.9 \mathrm{~g}$ /day between 1997 and $2009{ }^{49}$ whereas sodium intake was shown to have decreased from $6.6 \mathrm{~g} /$ day in 1997 to $4.7 \mathrm{~g}$ /day in $2009 .{ }^{50}$ The 2010 China Health and Nutrition Survey also showed a steady decline in sodium consumption from $6.8 \mathrm{~g} /$ day in 1991 to $5.9 \mathrm{~g} /$ day in 2000 and further down to $4.3 \mathrm{~g}$ /day in $2011 . .^{51}$

Based on 4-day dietary recalls, the INTERMAP study ${ }^{52}$ in the 1990 s found that as much as $75.8 \%$ of sodium intake among Chinese are from salt added during home cooking (Figure 1). New results had similar findings. The Chinese population derive over $80 \%$ of their sodium intake from salt added during the cooking process, which is in sharp contrast to the developed world where majority of population sodium intake originate from processed food. ${ }^{53}$ In Beijing, it was estimated that $90 \%$ of salt intake was from cooking and $<10 \%$ from processed food. ${ }^{54}$ These findings on sources of sodium intake have implications for designing the most effective salt reduction strategies.

In summary, salt/sodium intake in China is well above the recommended thresholds and physiological need. Variations of salt consumption level exist in China based on geographic location, ethnicity, gender, occupation, and age. Although there is a declining trend in the past few decades, salt intake

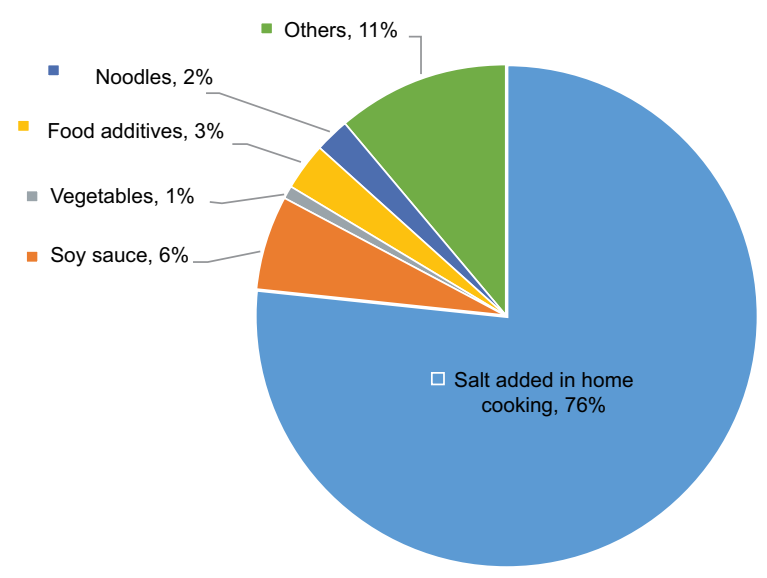

Figure I Dietary sources of sodium intake among Chinese residents. 
levels in China are high enough to warrant population-wide culturally tailored salt reduction schemes to address this public health issue, especially given the inclusion of salt reduction as one of the five highly cost-effective strategies for noncommunicable disease prevention and control by the WHO. ${ }^{29}$ The following sections briefly describe salt reduction initiatives in selected countries, followed by a more detailed account of such initiatives in China.

\section{Worldwide salt reduction initiatives}

As the pioneer of population-wide salt reduction initiatives, the UK established the Consensus and Action on Salt and Health (CASH) in 1996 aiming to encourage orchestrated salt reduction efforts between the government, food manufacturers, and consumers. ${ }^{55} \mathrm{CASH}$ initiated the National Salt Awareness Day in UK since 2000 and successfully lobbied the Food Standards Agency to implement a national salt reduction campaign with a target of $6 \mathrm{~g}$ /day by 2015 and a progressive reduction to a daily target of $3 \mathrm{~g}$ by $2025 .{ }^{55}$ Since the start of the program in $2003 / 2004$, over 7 years, there has been a $15 \%$ reduction in 24-h urinary sodium (from 9.5 to $8.1 \mathrm{~g} /$ day, $P<0.05$ ) and reduction in salt content of many processed food. ${ }^{56}$ The success of the UK's salt reduction initiatives can be attributed to the establishment of an action group with strong leadership and scientific credibility, setting progressive lower salt targets for different food categories with clear timeframes, collaboration with the food industry, clear nutritional labeling, and public awareness campaigns. ${ }^{56}$

Given that $75 \%-80 \%$ of sodium intake is from processed food or restaurants, ${ }^{57}$ the American government established the National Salt Reduction Initiative (NSRI) as a publicprivate partnership, aiming to reduce population salt intake through product reformulation. ${ }^{57}$ Similar to the UK, the US engaged in multifaceted salt reduction initiatives including setting different salt content targets in different food categories, introducing clear nutritional labeling, and initiating public awareness campaigns. ${ }^{57}$

The Australian government endorsed the WHO's action plan by pledging to achieve a $30 \%$ reduction in average population salt intake by 2025 . The average salt intake in Australia is $8-9 \mathrm{~g} /$ day with as much as $80 \%$ of salt intake from processed food. ${ }^{58}$ Government-led salt reduction strategies include the Food and Health Dialogue launched in 2011, which set voluntary salt targets for 11 food categories. Additionally, food manufacturers could adopt the Health Star Rating System to highlight the nutritional profiles of packaged food by displaying different number of stars in front labeling. ${ }^{58}$ A robust national monitoring mechanism is needed for Australia to achieve the target of $30 \%$ reduction by 2025 .

In developing countries like Malaysia, the government issued national policy targeting healthy eating and collaborated with nongovernmental organizations to promote dietary guidelines. The food industry is also engaged by the government to reformulate products containing less salt. ${ }^{59}$ South Africa sets a target of $<5 \mathrm{~g}$ of daily salt intake by 2020 . To achieve this, in 2013 the South African government passed legislation to mandatorily engage the food industry with manufacturing of products with lower salt content. Public awareness campaigns about salt intake were also launched. ${ }^{60}$

In 2005, the WASH was established based on the CASH program to implement similar salt reduction interventions around the world. To date, 527 members from 95 countries have joined the WASH movement. ${ }^{60}$ In 2012, the WHO set $5 \mathrm{~g}$ as the recommended daily salt intake to prevent and control hypertension. ${ }^{6} \mathrm{~A} 30 \%$ reduction in mean population salt intake by 2025 was included as one of the nine voluntary targets set by the World Health Assembly in 2013 to reduce behavioral and physiological risk factors. ${ }^{61}$

\section{Salt reduction in China}

On the basis of the search strategy and selection criteria described in the "Methods" section, we synthesized the salt reduction interventions in detail (Supplementary material C). A PRISMA selection flowchart can be found in Supplementary material D. We present our synthesis in five subsections, namely policy environment, national initiatives, regional initiatives, health awareness and health education, and research on low-sodium salt/salt substitute.

\section{Policy environment}

Commissioned by 15 Chinese ministries in 2012, the National Plan of the Prevention and Control of Chronic Diseases in China 2012-2015 sets a clear target of "reducing the Chinese population's daily salt intake below 9 grams by 2015." The Chinese Dietary and Nutritional Development Regulation 2014-2020 issued by the State Council in 2014 further stipulated "control of the salt and oil consumption" and "develop nutritional food low in sodium and fat" as key development goals. ${ }^{53}$

From January 1, 2013, all food manufacturers in China must affix labels to food products indicating the content of sodium, protein, fat, and carbohydrates under the Nutritional Labelling in Prepacked Food Legislation (GB28050-2011). 
The manufacturers of low-sodium salt products are subject to Industry Standard of Low Sodium Salt QB2019-2005 from $2005 .^{53}$ The most recent industry standard regulating the production of low-sodium salt NY/T 1040-2010 was stipulated by the Chinese Ministry of Agriculture in 2012 preceding over NY/T 1040-2006. ${ }^{62}$

A detailed description of salt reduction-related regulations in China can be found in Supplementary material E.

\section{National initiatives}

The China Healthy Lifestyle for All (CHLA) campaign was jointly launched in 2007 by the National Health and Family Planning Commission (formerly the Ministry of Health), the Office of National Patriotic Health Campaign Committee, and the Chinese Center for Disease Control and Prevention (China CDC). Its daily operations were managed by the China $\mathrm{CDC}$ to disseminate a number of healthy lifestyle messages including "daily salt intake should be $6 \mathrm{~g}$ or less." Using a multisector approach involving the private sector, health professionals, industries, schools, and community organizations, it disseminates health educational messages on recommended maximum intake of salt and promotes the uptake of healthy eating support tools (ie, salt restriction tools). The campaign utilizes mass media, educational program, billboards, and printed materials. By the end of 2011, a report showed that $40 \%$ of all Chinese counties had implemented the strategies. ${ }^{63}$

Later, the China Salt Reduction Initiative (2010-2020) initiated by the Chinese Centre of Disease Control sets a daily salt intake target at $6 \mathrm{~g}$ by 2020 and promotes the consumption of low-sodium, high-potassium salt, and salt substitutes. ${ }^{64}$

A cross-sectional survey was conducted in 2012 among 31,396 participants from 31 provinces to assess the efficacy of CHLA campaign. ${ }^{65}$ From each province, a county with exposure to the CHLA campaign (intervention group) was matched with another county with similar GDP and population without the CHLA exposure (control group). Only adults who have lived in the area for $>6$ months during the 12 months before the face-to-face survey date were included in the study. It was found that in urban sites, $49.2 \%$ of the intervention group use salt restriction spoon $(29.3 \%$ in control group, $P<0.01)$. In rural sites, uptake of salt restriction spoon is $28.4 \%$ among intervention group and $19.9 \%$ in control group $(P<0.01){ }^{65}$

Based on the same data, using intention-to-treat analysis via multilevel logistic regression, participants in "intervention" counties were found to be more likely to know the limit of salt (odds ratio: 3.14 ) and were more inclined to modify their consumption (odds ratio: 1.98) and to report a change in taste (odds ratio: 1.90). Such effect is not subject to income or education, whereas women and the elderly benefit disproportionately from the intervention. ${ }^{63}$

\section{Regional initiatives}

Regionally, the Beijing and Shanghai municipal governments distributed free salt restriction spoons to their residents in 2007 and 2008, respectively. The Shanghai government also disseminated the educational message of "target your daily salt intake to $6 \mathrm{~g}$ " accompanying the distribution of such spoons. ${ }^{53,66}$ The data from the 2010 Chinese National Chronic Disease and Risk Factor Surveillance suggested that salt restriction spoons were adopted by only $10.21 \%$ of Chinese population with the urban population being much more receptive to such practice compared to their rural counterparts. ${ }^{67}$

In 2009, the Beijing Municipal Government launched the "Healthy Beijing-Action Plan advancing population health (2009-2018)." ${ }^{\circ 8}$ Specifically, a target of $<10$ g/day was set for daily salt intake. The action plan also included the promotion of low-sodium salt to restaurants, institutional canteens/ cafeterias, and families. Subsidies were proposed to be given to food manufacturers to develop healthy food with low salt content. Shops and supermarkets were encouraged to set up designated area for food with less salt content to facilitate purchase among health-conscious customers. In 2010, the Beijing government also promoted the uptake of low-sodium salt substitute and strengthened the supply chain so that residents could purchase low-sodium salt from 28 supermarket chains. Residents received a bonus of $75 \mathrm{~g}$ of low-sodium salt and a salt restriction spoon both paid for by the government when they purchase $400 \mathrm{~g}$ of such product. ${ }^{53}$

However, a 2012 survey in the rural Shunyi District in Beijing found that only $41.4 \%$ of residents have heard of lowsodium salt and $30.4 \%$ are aware of its health benefits. Residents with lower income or less education were less receptive to such products. Main barriers of incorporating low-sodium salt into daily cooking included lack of awareness, difficulty of purchase, and lack of perceived health benefits. ${ }^{69}$

A 2012 survey conducted in the Shunyi district of Beijing found that using multistage randomized cluster sampling, $59.2 \%$ of the 1043 residents interviewed have used saltrestricted tools. ${ }^{69}$ A later study in the same area among 403 residents incorporating improved salt restriction tools accompanying health education found improved regular usage of such tools from $26.1 \%$ to $67.3 \%$ after a 6 -month intervention period..$^{70}$ Approximately $37.3 \%$ of subjects were able to correctly use the tools compared to $13.3 \%$ at baseline. Using the weighed food record method, intervention group 
also showed a $1.5 \mathrm{~g}$ /day lower salt intake compared to the control group after the intervention. Using the 24-h urinary excretion method, a study conducted in Beijing found that a 6-month intervention consisting of salt restriction spoon and health education resulted in $1.42 \mathrm{~g}$ reduction of daily salt intake in the intervention group compared to $0.28 \mathrm{~g}$ in control group..$^{71}$

The Shanghai Municipal Center for Disease Control and Prevention conducted evaluation of the Mass Campaign for Salt Control (mainly consisting of the distribution of salt-control spoons) and found that $41.15 \%$ of families still use the salt-control spoons during the 12 th month into intervention although the percentage decreased since the initial distribution in the 1 st month; $45.69 \%$ of the subjects have a daily salt intake of $\leq 6 \mathrm{~g}$ at the end of the intervention period, up from $37.24 \%$ at baseline $;{ }^{72}$ and $79.32 \%$ of residents have salt restriction-related knowledge after intervention, compared to $39.52 \%$ at baseline. ${ }^{73} \mathrm{TV}$ and popular science articles are the preferred ways for the residents to obtain saltrelated knowledge. The sample selected (>6000 residents in 2900 families) during the evaluation was also found to have low attrition rates. ${ }^{74}$

In the Minhang district of Shanghai, a 1-year intervention consisting of distribution of salt restriction tools accompanying health education materials was implemented among 371 residents in 160 families. Salt reduction awareness rate was found to improve from $88.1 \%$ to $95.4 \%$, and a significant increase $(P<0.01)$ was observed in people who use the tools to measure salt intake daily $(62.4 \%$ at 1 month into the intervention to $76.9 \%$ at 1 year after the intervention). ${ }^{75}$ In another similar study conducted in the Baoshan district of Shanghai in 2008, 2 years after the distribution of salt restriction spoons to 160 families, ${ }^{66}$ awareness rate of relationship between salt and health improved from $58.5 \%$ at baseline to $79.2 \%$. Approximately $68.4 \%$ of subjects engaged in persuading family members to reduce adding cooking salt at the end of the intervention, indicating a $8 \%$ increase compared to baseline $(P<0.05)$ although a curvilinear pattern (increase in first year followed by a minor decrease in second year) was found in the improvements above.

Residents in Shandong (the second most populous province in China) have a strong preference for salty food and a 2011 cross-sectional survey estimated that over $23.4 \%$ of residents in Shandong had hypertension. ${ }^{76}$ In 2011, the provincial government of Shandong and the Ministry of Health launched the Shandong Province-Ministry of Health Action on Salt Reduction and Hypertension (SMASH) with a target to reduce daily salt intake to $10 \mathrm{~g}$ /day by 2015 and to increase awareness and control of hypertension among adults in Shandong. ${ }^{76}$ This is the first large-scale provincial government-led intervention targeting population salt reduction in China; and the midterm evaluation showed the promising result of a reduction in the per capita seasoning salt intake from 12.5 to $11.58 \mathrm{~g} /$ day among adults aged 18-69 years. ${ }^{77}$ Furthermore, one modeling study based on SMASH data suggested that if the sodium consumption was reduced from the 2011 baseline level to 3.5 or $4.0 \mathrm{~g} /$ day, in Shandong province alone, 19,800 (13,800-24,900) deaths or 14,400 deaths $(10,100-19,500)$ from cardiovascular diseases could be prevented each year, respectively. ${ }^{78}$

Common barriers in the uptake of salt restriction tools include lack of education on using the tools, inability to correctly use the tools, and inconvenience. Chen et al examined the factors affecting the adoption of such spoons and concluded that especially among young people with risk of hypertension, population with low education level, and rural population, enhanced intervention consisting of education on salt reduction benefits and right usage of salt-restriction spoon is required. ${ }^{79}$

\section{Health awareness and health education}

There has been many salt reduction strategies with a public education focus to improve the awareness of risk associated with diets rich in salt and encourage the Chinese population to follow a healthy diet. ${ }^{80} \mathrm{~A}$ randomized cluster sample conducted on 96,916 Chinese adults in 2010 found that the unweighted rate of the awareness, willingness, and behavior toward salt reduction among Chinese adults were $63.0 \%$, $84.5 \%$, and $44.5 \%$, respectively. ${ }^{81}$ After being weighted, significant inverse relationship exists between age and awareness rate as well as willingness rate. Urban residents demonstrated significantly higher awareness, willingness, and behavior rates than their rural counterparts. Women also demonstrated significantly higher willingness rate and behavior rate than men.

From 1974 to 1998 , a large-scale worksite-based intervention was implemented among 110,000 employees in a large iron and steel manufacturer in Beijing. ${ }^{82}$ Eight simultaneously parallel reference groups in other provinces outside Beijing were set up based on population surveillance data. With the focus of primary prevention of cardiovascular diseases and hypertension, the comprehensive intervention included health education, health promotion, dietary adjustment focusing on salt reduction, and other lifestyle risk factor management. ${ }^{83} \mathrm{BP}$ and body mass index (BMI) were found to have decreased relatively in the intervention group 
during 1974-1998, compared to significantly increased BP and BMI in majority of the eight reference groups at different provinces in China. ${ }^{83}$

The SchEdu Salt study involved 584 school children from 28 primary schools to deliver salt reduction messages to their families in Changzhi, Shanxi Province. Children from 14 schools in the intervention group received education on the adverse effects of salt and the method to reduce salt intake during the schools' usual health education lessons. The other 14 schools in the control group did not receive such salt reduction-focused education. ${ }^{84}$ The intervention lasted one semester long (3.5 months) and saw on average a $2.9 \mathrm{~g}$ /day reduction among adults in daily salt intake compared to the control group $(P<0.001)$, whereas for children, the mean effect was $1.9 \mathrm{~g} /$ day $(P<0.001) .{ }^{85}$ The mean effect on SBP was $-2.3 \mathrm{mmHg}$ in adults and $-0.8 \mathrm{mmHg}$ in children compared to their counterparts in the control group. ${ }^{85}$

\section{Research on low-sodium salt/salt substitute}

For people accustomed to a salty diet, education and saltrestricted tools alone are insufficient to prompt long-term behavior change. To reduce population sodium intake, it is an easier option for people to substitute normal salt with low-sodium salt with a marginally different taste but much lower sodium content. Predominantly, low-sodium salt has been manufactured with part of the sodium chloride replaced by potassium chloride. The proportion of potassium chloride ranges from $30 \%$ to $50 \%$ and magnesium sulfate is sometimes added to balance the bitterness of potassium chloride. ${ }^{86}$

Salt substitute has been available in the Chinese market for decades, and its taste is generally acceptable. ${ }^{87}$ The safety and long-term use of potassium-enriched salt was evaluated among 112 volunteers during a 1-year intervention. Health education and potassium-enriched salt $(\mathrm{NaCl}: \mathrm{KCl}$ $=1: 1$ ) were provided to participants every 3 months. The spot urinary ratio of sodium to potassium was significantly reduced, and no side effect on the liver and kidney function was found. Although salt substitutes are generally safe as no serious adverse effects were reported in decades of sales, further study is still needed given the sample size and intervention period of this study. ${ }^{88}$

The Industry Standard on Green Food-Edible Salt NY/T 1040-2012 stipulated by the Chinese Ministry of Agriculture superseded its predecessor NY/T 1040-2006 in 2012. The new Standard removed the limitation on magnesium content and set the required range for sodium chloride at $70 \% \pm 10 \%$ and potassium chloride at $24 \% \pm 10 \%$ in low-sodium salt products. $^{62}$
The effects of salt substitute on BP have been investigated in several trials in China. The China Salt Substitute Study was a randomized controlled trial conducted in 2004 among 608 high cardiovascular-risk individuals (mean age $=60$ years) in six rural Northern areas. The study found that the intervention group using the standard salt substitute $(65 \% \mathrm{NaCl}, 25 \% \mathrm{KCl}$, $10 \% \mathrm{MgSO}_{4}$ ) benefited from reduced arterial stiffness ${ }^{89}$ in addition to significantly reduced peripheral and central SBP over a 12-month intervention period compared to the control group using regular salt $(100 \% \mathrm{NaCl}) .{ }^{89,90}$

Zhou et al further assessed the impact of salt substitution on BP of rural northern Chinese in two consecutive studies. ${ }^{91,92}$ The first study conducted among 200 families with a 2-year intervention period found that in a double-blinded, randomized controlled trial, salt substitute $(65 \% \mathrm{NaCl}, 25 \%$ $\mathrm{KCl}, 10 \% \mathrm{MgSO}_{4}$ ) lowered both SBP and diastolic blood pressure (DBP) among normotensive subjects and lowered only SBP among hypertensive subjects. ${ }^{91}$ The follow-up study extended the intervention to 200 families in five villages in Northern China during a 3-year double-blinded, randomized controlled trial with the same salt substitute and found significant decrease in both SBP and DBP from baseline to the end of 36-month follow-up in the intervention group compared with the control group using normal salt (100\% sodium chloride group), demonstrating the long-term benefit of salt substitute on lowering BP among rural Northern Chinese. ${ }^{52}$

The same salt substitute was used in a single-blinded, randomized controlled trial targeting Tibetans with known hypertension (SBP >140 mmHg). After a 3-month intervention, $19.2 \%$ of patients in the intervention group had their BP under control compared to only $8.8 \%$ in control group $(P=0.027)$. Net reduction in SBP/DBP in the intervention group in comparison with the control group was $8.2 / 3.4 \mathrm{mmHg}$ (based on per protocol analysis) and 7.6/3.5 mmHg (based on intention-to-treat analysis).

Another quasi-experiment conducted on 411 adults in rural Shandong province found that using the commercially available low-sodium and high-potassium salt substitute results in decreased BP of both normotensive and hypertensive population. Both the groups also demonstrated decreased urinary sodium and increased urinary potassium. ${ }^{93}$ Targeting 325 hypertensive adolescents and 978 of their families in a rural Chinese community, a salt substitute containing potassium and calcium was found to be as effective as sodium restriction in reducing the BP of hypertensive adolescents and their families. ${ }^{94}$

In 2011, the China Rural Health Initiative was conducted as a large-scale, cluster-randomized trial in five Northern 
Chinese provinces comprising 120 villages in 120 townships. The intervention included community-based health education and promotion of salt substitutes $(65 \% \mathrm{NaCl}, 25 \% \mathrm{KCl}, 10 \%$ $\left.\mathrm{MgSO}_{4}\right) .{ }^{95}$ Salt substitutes were made available in village convenience stores for sale in 60 villages in the intervention group. Half of these 60 villages were randomly selected to receive subsidy to make the more expensive salt substitutes cost the same as regular salt. Twenty four-hour urinary samples showed reduced daily sodium intake and increased daily potassium intake among participants in intervention groups. ${ }^{96}$ The combination of salt substitute and health education was also found to reduce albuminuria among 1903 rural Chinese in this cluster randomized trial $(P<0.01) .{ }^{97}$

Furthermore, the China Salt Substitute and Stroke Study ${ }^{23}$ (ClinicalTrials.gov identifier: NCT 02092090) is an ongoing large-scale cluster randomized trial targeting 600 villages and 21,000 participants across five Northern Chinese provinces and Tibet with an expected completion date of 2019. The study will define the effects of a salt substitute-based sodium reduction strategy on the outcome of stroke and mortality.

\section{Recommendations and conclusion}

Excessive salt intake among the Chinese population is a major contributor to the high prevalence of hypertension and cardiovascular diseases. In China, population-based salt reduction strategy should be adopted for the prevention and control of cardiovascular diseases and other health benefits. Based on the review, the following recommendations for effective population-wide salt reduction were proposed. The full impact and best approach in implementing these recommendations need to be further studied.

\section{Chinese Salt Reduction Task Force}

The Chinese government has an unequivocal role in reducing its population salt intake. Historically, the Chinese Center of Disease Control is responsible for the planning and implementation of salt reduction programs with the Ministry of Health playing a steering role. ${ }^{53}$ Other sectors including the Ministry of Education, the Ministry of Communication, the Quality Control Bureau, as well as the State Food and Drug Administration should take on more active roles in order to create a nationwide supportive policy environment for salt reduction.

Chinese government also needs to align the interests of different stakeholders including the food industry, salt manufacturers, consumers, and academic institutions to efficiently push its salt reduction agenda forward. We recommend the establishment of Chinese Salt Reduction Task Force comprising reputable individuals from various government ministries, industries, and academia. This task force can work alongside the fledgling China Salt Reduction Initiative to spearhead and coordinate nation-wide salt reduction efforts. Clear and achievable goals should be set early on as well as monitoring systems to evaluate progress toward the goals. Members of the working group should meet regularly to discuss the progress and challenges related to salt reduction in their respective community, brainstorming innovative salt reduction campaigns, and acting as think tank for the Chinese government in salt reduction-related matters.

\section{Health education and promotion}

Population sodium reduction cannot be achieved without raising awareness among the general public given the majority of salt intake among Chinese come from salt added during the cooking process. In addition to raising awareness through worksite-based interventions, salt reduction education should also be delivered targeting pregnant women, students, and teachers, so that children and teenagers could benefit from low-sodium diet from an early age. The existing communication channels of the various government entities should be fully utilized to raise awareness among their respective target communities.

Salt reduction campaigns should nonetheless avoid the absolute vilification of salt. Health messages shall emphasize that it is the excessive intake of salt ( $>5-6 \mathrm{~g}$ /day) that is harmful to health. Besides messages on the harm of high salt intake and benefits of moderate intake, the importance of self-measurement of salt intake as well as practical measures to reduce salt intake should also be emphasized. It is widely recognized that health education efforts are more effective when promoted in combination with other tangible strategies such as affordable and useful tools and products (eg, salt substitutes).

\section{Food industry legislation and food labeling}

In a market economy, food industries are mainly motivated by profit and could therefore resist any voluntary changes to product reformulation including sodium reduction in fear of losing customers. Industrial regulations should stipulate salt targets for different food categories in order to reduce the sodium content of manufactured food.

Similar to the UK and Australia, China could also introduce food labeling prominently displaying the health rating of each food based on sodium and other key nutritional content. As health awareness increases, low-sodium products will gain more popularity. Standardized nutritional profile (including sodium content) of common Chinese dishes should be researched and disseminated, so that the general 
public could conveniently estimate their sodium intake from common dishes.

\section{Salt restriction tools and salt substitutes}

The distribution of salt restriction tools should be scaled up to the fast food and restaurant industry and more provinces to curb salt added while cooking. New technologies such as smartphone-based applications and other mobile health tools are worth further development, evaluation, and promotion. Salt substitute with lower sodium has great potential in reducing population salt intake especially in northern and rural China where the preference for salty food is deep-rooted and far-reaching. For diagnosed hypertensive patients or those with cardiovascular diseases, a subsidy should be included in their medical insurance for the purchase of low-sodium salt substitute given its higher price compared to regular salt. In addition, government subsidy for salt substitute manufacturing and purchase is potentially beneficial for population health.

Community-based primary health care centers should play a larger role in the distribution of salt restriction tools and salt substitute, so that larger high-risk population could be reached in a sustainable manner. Primary health care centers in China, although still under development, should be further integrated in the NSRI for its sustainability and costeffectiveness, especially for its potential to be the one-stop shop for receiving health education, salt restriction tools, and low-sodium salt substitute to the high-risk target populations and the general public.

China is a country with excessive salt intake among the majority of the population with average intake doubling the recommended upper limit. Even people who typically have reservations on universal salt reduction strategy reach consensus about its value for countries like China. Orchestrated efforts are required from the Chinese government, food industries, health professionals, and the general public to achieve the long-term salt reduction goal in China. To quote an ancient Chinese poet Qu Yuan, "The way ahead is long and has no ending; yet high and low we'll search with our will unbending".

\section{Disclosure}

The authors report no conflicts of interest in this work.

\section{References}

1. Murray CJL, Lopez AD. Measuring the global burden of disease. $N$ Engl J Med. 2013;369(5):448-457.

2. GBD 2013 Mortality and Causes of Death Collaborators. Global, regional, and national age\& sex specific all-cause and cause-specific mortality for 240 causes of death, 1990-2013: a systematic analysis for the Global Burden of Disease Study 2013. Lancet. 2015;385(9963):117-171.
3. Lim SS, Vos T, Flaxman AD, et al. A comparative risk assessment of burden of disease and injury attributable to 67 risk factors and risk factor clusters in 21 regions, 1990-2010: a systematic analysis for the Global Burden of Disease Study 2010. Lancet. 380(9859):2224-2260.

4. IHME. China all ages DALYs (thousands) and age-standardised DALY rates (per 100000 ) with $95 \%$ UI for 306 causes, both sexes combined, in 1990, 2005, and 2013, with median percent change; 2015. Available from: http://ghdx.healthdata.org/global-burden-disease-study2013-gbd-2013-data-downloads. Accessed July 4, 2016.

5. Liu Y, Li H, Hong S, Yin X. Salt reduction and hypertension in China: a concise state-of-the-art review. Cardiovasc Diagn Ther. 2015;5(3):191-196.

6. World Health Organization. A Global Brief on Hypertension: Silent Killer, Global Public Health Crisis. Geneva, Switzerland: WHO; 2013.

7. Ambard L, Beaujard E. Causes de l'hypertension arterielle. Arch Gen Med. 1904;1:520-533.

8. Aburto NJ, Ziolkovska A, Hooper L, Elliott P, Cappuccio FP, Meerpohl JJ. Effect of lower sodium intake on health: systematic review and metaanalyses. BMJ. 2013;346:f1326.

9. Li XY, Cai XL, Bian PD, Hu LR. High salt intake and stroke: metaanalysis of the epidemiologic evidence. CNS Neurosci Ther. 2012; 18(8):691-701

10. Peng YG, Li W, Wen XX, Li Y, Hu JH, Zhao LC. Effects of salt substitutes on blood pressure: a meta-analysis of randomized controlled trials. Am J Clin Nutr. 2014;100(6):1448-1454.

11. Suckling RJ, He FJ, Macgregor GA. Altered dietary salt intake for preventing and treating diabetic kidney disease. Cochrane Database Syst Rev. 2010;(12):Cd006763.

12. Graudal NA, Hubeck-Graudal T, Jurgens G. Effects of low sodium diet versus high sodium diet on blood pressure, renin, aldosterone, catecholamines, cholesterol, and triglyceride. Cochrane Database Syst Rev. 2011;(11):Cd004022.

13. He FJ, Li J, Macgregor GA. Effect of longer term modest salt reduction on blood pressure: Cochrane systematic review and meta-analysis of randomised trials. BMJ. 2013;346:f1325.

14. He FJ, MacGregor GA. Effect of modest salt reduction on blood pressure: a meta-analysis of randomized trials. Implications for public health. J Hum Hypertens. 2002;16(11):761-770.

15. Kawasaki T, Delea CS, Bartter FC, Smith H. The effect of highsodium and low-sodium intakes on blood pressure and other related variables in human subjects with idiopathic hypertension. Am J Med. 1978;64(2):193-198.

16. Dumler F. Dietary sodium intake and arterial blood pressure. J Renal Nutr. 2009;19(1):57-60.

17. McMahon EJ, Campbell KL, Bauer JD, Mudge DW. Altered dietary salt intake for people with chronic kidney disease. Cochrane Database Syst Rev. 2015;(2):Cd010070.

18. Ettehad D, Emdin CA, Kiran A, et al. Blood pressure lowering for prevention of cardiovascular disease and death: a systematic review and meta-analysis. Lancet. 2016;387(10022):957-967.

19. Mente A, O'Donnell M, Rangarajan S, et al. Associations of urinary sodium excretion with cardiovascular events in individuals with and without hypertension: a pooled analysis of data from four studies. Lancet. 2016;388(10043):465-475.

20. He FJ, MacGregor GA. Hypertension: salt: flawed research should not divert actions to reduce intake. Nat Rev Nephrol. 2016;12(9):514-515.

21. Cogswell ME, Mugavero K, Bowman BA, Frieden TR. Dietary sodium and cardiovascular disease risk - measurement matters. $N$ Engl J Med. 2016;375(16):580-586.

22. World Action on Salt and Health. WASH \& CASH response to Publication in the Lancet by Mente et al on salt and blood pressure; 2016. Available from: http://www.worldactiononsalt.com/news/saltnews/2016/177218.html. Accessed August 7, 2016.

23. The George Institute. China Salt Substitute and Stroke Study (SSaSS); 2014. Available from: https://clinicaltrials.gov/ct2/show/NCT02092090. Accessed July 4, 2016. 
24. Zhao L, Stamler J, Yan LL, et al. Blood pressure differences between northern and southern Chinese: role of dietary factors: the International Study on Macronutrients and Blood Pressure. Hypertension. 2004;43(6): 1332-1337.

25. Wu Y, Lu C, Gao R, Yu J, Lie G. Nation-wide hypertension screening in China during 1979-1980. Chin Med J. 1982;95:101-108.

26. Wang X, Bots ML, Yang F, Hoes AW, Vaartjes I. Prevalence of hypertension in China: a systematic review and meta-regression analysis of trends and regional differences. J Hypertens. 2014;32(10):1919-1927.

27. Chen W, Gao R, Liu L, et al. A summary of China Cardiovascular Diseases Report 2013. Chinese Circulation J. 2014;29(7):487-491.

28. Wu Z, Yao C, Zhao D, et al. Sino-MONICA project: a collaborative study on trends and determinants in cardiovascular diseases in China, Part I: morbidity and mortality monitoring. Circulation. 2001;103(3) 462-468.

29. Beaglehole R, Bonita R, Horton R, et al. Priority actions for the noncommunicable disease crisis. Lancet. 2011;377(9775):1438-1447.

30. Mozaffarian D, Fahimi S, Singh GM, et al. Global Sodium Consumption and Death from Cardiovascular Causes. $N$ Engl J Med. 2014;371(7):624-634.

31. Wang M, Moran AE, Liu J, et al. Projected impact of salt restriction on prevention of cardiovascular disease in China: a modeling study. PLoS One. 2016;11(2):e0146820.

32. McLean RM. Measuring population sodium intake: a review of methods. Nutrients. 2014;6(11):4651-4662.

33. Liu L, Ikeda K, Yamori Y. Inverse relationship between urinary markers of animal protein intake and blood pressure in Chinese: results from the WHO Cardiovascular Diseases and Alimentary Comparison (CARDIAC) Study. Int J Epidemiol. 2002;31(1):227-233.

34. Liu L, Liu L, Ding Y, et al. Ethnic and environmental differences in various markers of dietary intake and blood pressure among Chinese Han and three other minority peoples of China: Results from the WHO Cardiovascular Diseases and Alimentary Comparison (CARDIAC) Study. Hypertens Res. 2001;24(3):315-322.

35. Stamler J, Elliott P, Dennis B, et al. INTERMAP: background, aims, design, methods, and descriptive statistics (nondietary). J Hum Hypertens. 2003;17(9):591-608.

36. Zhang Y, Wang M, Shi J, et al. Prevalence, awareness, treatment and control of hypertension and sodium intake in Jiangsu Province, China: a baseline study in 2014. BMC Public Health. 2016;16(1):56.

37. Zhang J, Yan L, Tang J, et al. Estimating daily salt intake based on $24 \mathrm{~h}$ urinary sodium excretion in adults aged 18-69 years in Shandong, China. BMJ Open. 2014;4(7):e005089-e005089.

38. Xu J, Wang M, Chen Y, et al. Estimation of salt intake by 24-hour urinary sodium excretion: a cross-sectional study in Yantai, China. BMC Public Health. 2014;14:136.

39. Ma G. The salt consumption of residents in China. Chinese J Prev Control Chronic Dis. 2008;16(4):214-217.

40. Hipgrave D, Chang S, Li X, et al. Salt and sodium intake in China. JAMA. 2016;315(7):703-705.

41. Zhao F, Zhang P, Zhang L, et al. Consumption and sources of dietary salt in family members in Beijing. Nutrients. 2015;7(4):2719-2730.

42. Chen J, Zhang R, Zhang H. Dietary intake and nutrition status of urban residents in Zhejiang province. Chinese J Public Health. 2011;2: 170-173.

43. Qi X, Wang Z, Jiang M, Cheng G. Investigation on salt intake of residents in Sanmen county. Zhejiang J Prev Med. 2015;03:281-282.

44. Hu M, Chen Z, Lin S, Lin X, Wang M, Wu J. Investigation and analysis on the salt intake of residents in Fujian province. Chinese $J$ Control Endemic Dis. 2010;06:435-437.

45. Li S, Wen J, Deng X. Status and change trend of food consumption and nutrients intake in urban population of Guangdong province. Zhong Guo Gong Gong Wei Sheng. 2014;09:1109-1112.

46. Yin L, Liu H, Liu J, Guan X. Dietary status and related influence factors of adults in Hunan province. Pract Prev Med. 2015;01:94-96.

47. Yan L, Xu JW, Zhang M. Analysis of self-reported daily salt intake per capital of residents households in China, 2010. Chinese J Health Educ. 2014;30(5):387-389
48. Liu Z. Dietary sodium and the incidence of hypertension in the chinese population: a review of nationwide surveys. Am J Hypertens. 2009;22(9):929-933.

49. Liu A. Chinese adult's dietary patterns and their relationship with hypertension among residents in nine provinces (1997-2009) [dissertation]. Beijing: Chinese Center of Disease Control; 2011.

50. Du S, Neiman A, Batis C, et al. Understanding the patterns and trends of sodium intake, potassium intake, and sodium to potassium ratio and their effect on hypertension in China. Am J Clin Nutr. 2014;99(2):334-343.

51. Zhai FY, Du SF, Wang ZH, Zhang JG, Du WW, Popkin BM. Dynamics of the Chinese diet and the role of urbanicity, 1991-2011. Obes Rev. 2014;15:16-26.

52. Okuda N, Stamler J, Brown IJ, et al. Individual efforts to reduce salt intake in China, Japan, UK, USA: what did people achieve? The intermap population study. J Hypertens. 2014;32(12):2385-2392.

53. Xu JW. Study on Salt Reduction Strategy for China. [dissertation] Bejing, China: China Center of Diseases Control; 2014.

54. Zhao F, Zhang P, Zhang L, et al. Consumption and sources of dietary salt in family members in Beijing. Nutrients. 2015;7(4):2719-2730.

55. CASH Consensus Action on Salt \& Health. Achievements; 2016. Available from: http://www.actiononsalt.org.uk/about/aims/31934.html. Accessed July 13, 2016.

56. He FJ, Brinsden HC, MacGregor GA. Salt reduction in the United Kingdom: a successful experiment in public health. J Hum Hypertens. 2014;28(6):345-352.

57. Reeve B, Magnusson R. Food reformulation and the (neo)-liberal state: new strategies for strengthening voluntary salt reduction programs in the UK and USA. Public Health. 2015;129(4):351-363.

58. Webster J, Trieu K, Dunford E, et al. Salt reduction in Australia: from advocacy to action. Cardiovasc Diagn Ther. 2015;5(3):207-218.

59. Batcagan-Abueg AP, Lee JJ, Chan P, Rebello SA, Amarra MS. Salt intakes and salt reduction initiatives in Southeast Asia: a review. Asia Pacific J Clin Nutr. 2013;22(4):490-504.

60. WASH World Action on Salt \& Health; 2016. Available from: http:// www.worldactiononsalt.com/. Accessed 13 July, 2016.

61. World Health Organization. NCD global monitoring framework; 2014. Available from: http://www.who.int/nmh/global_monitoring framework/en/. Accessed July 10, 2016.

62. Chinese Ministry of Agriculture. NYT1040-2012 Green Food-Edible Salt; 2013. Available from: http://www.tsinfo.js.cn/inquiry/gbtdetails. aspx?A100=NY/T\%201040-2012. Accessed September 10, 2016.

63. Zhang J, Astell-Burt T, Seo DC, et al. Multilevel evaluation of 'China Healthy Lifestyles for All', a nationwide initiative to promote lower intakes of salt and edible oil. Prev Med. 2014;67:210-215.

64. Chinese Center of Disease Control. China Salt Reduction Initative (2010-2020); 2010. Available from: http://www.docin.com/p977861860.html. Accessed August 4, 2016.

65. Li Y, Zhang J, Shi XM, Liang XF. Zhongguo chengxiang jumin 2012 nian quanmin jiankang shenghuo fangshi xingdong shishi xiaoguo diaocha [A cross-sectional survey on the efficacy of "China Healthy Lifestyle for All” in 2012]. Zhong Guo Liu Xing Bing Xue Za Zhi. 2013;34(9):869-873. Chinese.

66. Cai Z, Chen T. Effect assessment of salt reduction intervention among residents in Baoshan district, Shanghai. Chinese J Health Educ. 2011;27(8):571-573.

67. Xu JW, Yan LX, Zhang M, et al. Investigation on the coverage of salt control spoon and oil control pot among Chinese resident households in 2010. Chinese J Health Educ. 2014;30(5):390-392.

68. Beijing Municipal People's Government. Healthy Beijing-Action Plan advancing population health (2009-2018); 2009. Available from: http:// www.wsjsw.gov.cn/wsj/n422/n424/u1ai139715.html. Accessed July 3, 2016.

69. You K, Zhang Y, Wang JL, Zhao HY. Investigation of the use of lowsodium salt and salt-restriction tools among residents in Shunyi District, Beijing. Chin J Public Health. 2013;29(10):1448-1449.

70. Yang S, Zhang X, He C, Chan J, Sun X. Analysis of the effect of salt reduction initatives in Shunyi District, Beijing. China J Prev Contr Chron Dis. 2015;23(12):918-921. 
71. Chen J, Tian Y, Liao Y, et al. Salt-restriction-spoon improved the salt intake among residents in China. PLoS One. 2013;8(11):e78963.

72. Wang Y, Shi Y, Wu C, et al. Evaluation on a mass campain for salt control in Shanghai: change in salt consumption. J Environ Occup Med. 2015;32(1):38-42.

73. Wang X, Li M, Ding J, et al. Evaluation on a mass campaign for salt control in Shanghai: knowledge and behavior improvements on restricted salt usage. J Environ Occup Med. 2015;32(1):22-26.

74. Ding J, Yu H, L M, et al. Evaluation on a mass campaign for salt control in Shanghai: sampling validity of a large population-based study. J Environ Occup Med. 2015;32(1):27-31.

75. Mei KW, Wei MQ, Wang J. KABP investigation of residents on salt control in Minhang district of Shanghai and evalution on the intervention effectiveness of dispatching salt-control spoons. China Health Educ J. 2010;26(9):655-657.

76. Bi Z, Liang X, Xu A, et al. Hypertension prevalence, awareness, treatment, and control and sodium intake in Shandong Province, China: baseline results from Shandong-Ministry of Health Action on Salt Reduction and Hypertension (SMASH), 2011. Prev Chron Dis. 2014;11:E88.

77. Neiman A, Levings J, Ying C. Two initiatives worth their salt: reducing sodium intake in Shandong, China and Philadelphia; 2015. Available from: http://www.cdc.gov/globalhealth/stories/reducing_sodium_2015. html. Accessed November 28, 2016.

78. Liu S, Cai X, Hong Y, Zhou M. Cardiovascular disease deaths and high sodium consumption in Shandong province and China: a modelling analysis. Lancet. 2015;386:S80.

79. Chen J, Liao Y, Li Z, et al. Determinants of salt-restriction-spoon using behavior in China: application of the health belief model. PLoS One. 2013;8(12):e83262.

80. Xi B, Hao Y, Liu F. Salt reduction strategies in China. Lancet. 2014; 383(9923):1128.

81. Xu J, Yan L, Chen X, et al. 2010 nian woguo chengnianren jianyan zhishi, taidu he xingwei qingkuang fenxi [Investigation on knowledge, attitude and behavior of salt reduction in Chinese adults, 2010]. Zhonghua $Y u$ Fang Yi Xue Za Zhi. 2014;48(5):350-354. Chinese.

82. Chen J, Wu X, Gu D. Hypertension and cardiovascular diseases intervention in the capital steel and iron company and Beijing Fangshan community. Obes Rev. 2008;9(Suppl 1):142-145.

83. Wu XG, Gu DF, Wu YF, et al. Shoudu gangtie gongsi renqun xinxueguanbing 24 nian ganyu xiaoguo pingjia [An evaluation on effectiveness of worksite-based intervention for cardiovascular disease during 1974-1998 in capital iron and steel company of Beijing]. Zhonghua Yu Fang Yi Xue Za Zhi. 2003;37(2):93-97. Chinese.

84. He FJ, Wu Y, Ma J, et al. A school-based education programme to reduce salt intake in children and their families (School-EduSalt): protocol of a cluster randomised controlled trial. BMJ Open. 2013; 3(7): $\mathrm{e} 0033888$.
85. He FJ, Wu Y, Feng XX, et al. School based education programme to reduce salt intake in children and their families (School-EduSalt): cluster randomised controlled trial. BMJ. 2015;350:h770.

86. Yan L, Li Y, Wu YF. Jianyan fangkong gaoxueya: zhongguo renqun de zhengju ji yingdui celue jianyi [Reducing salt intake for hypertension control: related evidence and recommendation on strategies in China]. Zhonghua Liu Xing Bing Xue Za Zhi. 2011;32(12):1188-1192. Chinese.

87. Li N, Prescott J, Wu Y, et al. The effects of a reduced-sodium, highpotassium salt substitute on food taste and acceptability in rural northern China. Br J Nutr. 2009;101(07):1088-1093.

88. Ma Y, Li C, Huang X, You H, Zhang H. Safety evaluation of enrichedpotassium salt in Chinese population. Int J Cardiol. 2011;152:S43.

89. Hu J, Jiang X, Li N, et al. Effects of salt substitute on pulse wave analysis among individuals at high cardiovascular risk in rural China: a randomized controlled trial. Hypertens Res. 2009;32(4):282-288.

90. The China Salt Substitute Study Collaborative Group. Salt substitution: a low-cost strategy for blood pressure control among rural Chinese. A randomized, controlled trial. J Hypertens. 2007;25(10):2011-2018.

91. Zhou B, Wang HL, Wang WL, Wu XM, Fu LY, Shi JP. Long-term effects of salt substitution on blood pressure in a rural North Chinese population. J Hum Hypertens. 2013;27(7):427-433.

92. Zhou B, Webster J, Fu L-Y, et al. Intake of low sodium salt substitute for 3 years attenuates the increase in blood pressure in a rural population of North China - a randomized controlled trial. Int J Cardiol. 2016;215:377-382.

93. Zhang GH, Ma JX, Guo XL, et al. [Field observation on the effect of low-sodium and high-potassium salt substitute on blood pressure in the rural community-based population in China]. Chinese J Epidemiol. 2011;32(9):859-863.

94. Mu J, Liu Z, Liu F, Xu X, Liang Y, Zhu D. Family-based randomized trial to detect effects on blood pressure of a salt substitute containing potassium and calcium in hypertensive adolescents. Am J Hypertens. 2009;22(9):943-947.

95. Li N, Yan L, Niu W, et al. China rural health initiative - sodium reduction study: the effects of a community-based sodium reduction program on 24hr urinary sodium and blood pressure in rural China. Circulation. 2013;128(24):2707.

96. The George Institute for Global Health China. Study identifies a new community-based strategy to reduce population sodium intake in rural China; 2013. Available from: http://www.georgeinstitute.org.cn/media-releases/ study-identifies-a-new-community-based-strategy-to-reduce-population-sodium-intake-in?utm_source=George+Institute+External+Email +List\&utm_campaign=0783bfc986-TGI_eNewsletter_RADAC\&utm_ medium=email\&utm_term=0_0972953fbb-0783bfc986-95170073. Accessed August 22, 2016.

97. Jardine M, Li N, Ninomiya T, et al. Dietary sodium reduction reduced albuminuria in 1,903 rural chinese: a cluster randomised trial. Nephrology. 2014;19:28.
Risk Management and Healthcare Policy

\section{Publish your work in this journal}

Risk Management and Healthcare Policy is an international, peer-reviewed, open access journal focusing on all aspects of public health, policy, and preventative measures to promote good health and improve morbidity and mortality in the population. The journal welcomes submitted papers covering original research, basic science, clinical and epidemiological

\section{Dovepress}

studies, reviews and evaluations, guidelines, expert opinion and commentary, case reports and extended reports. The manuscript management system is completely online and includes a very quick and fair peerreview system, which is all easy to use. Visit http://www.dovepress.com/ testimonials.php to read real quotes from published authors. 